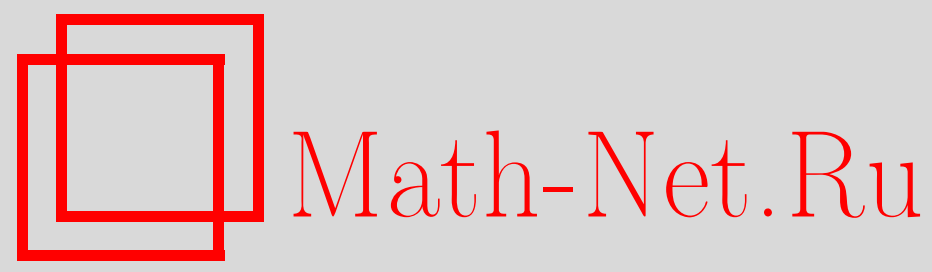

А. М. Горцев, Л. А. Нежельская, Асинхронный дважды стохастический поток с инициированием лишних событий, Дискрет. матем., 2011, том 23, выпуск 2, 59-65

DOI: https://doi.org/10.4213/dm1141

Использование Общероссийского математического портала Math-Net.Ru подразумевает, что вы прочитали и согласны с пользовательским соглашением http://www . mathnet.ru/rus/agreement

Параметры загрузки:

IP: 3.95 .254 .165

26 апреля 2023 г., 14:35:20 


\title{
Асинхронный дважды стохастический поток с инициированием лишних событий
}

\author{
(c) 2011 г. А. М. Горцев, Л. А. Нежельская
}

\begin{abstract}
Рассматривается асинхронный дважды стохастический поток с инициированием лишних событий (обобщенный асинхронный поток), являющийся математической моделью информационных потоков, функционирующих в информационно-вычислительных сетях, телекоммуникационных системах, компьютерных сетях связи и т. п. Исследуется стационарный режим функционирования потока. Находится плотность вероятностей $p(\tau)$ длительности интервала между событиями потока, а также совместная плотность вероятностей $p\left(\tau_{1}, \tau_{2}\right)$ длительностей двух смежных интервалов. Показывается, что обобщенный асинхронный поток в общем случае является коррелированным потоком. Находятся условия, при которых поток становится рекуррентным либо вырождается в простейший.

Работа выполнена в рамках задания Федерального агентства по образованию на проведение научных исследований в Томском государственном университете на 20062007 годы, задание 1.22.06.
\end{abstract}

\section{1. Введение}

В связи с интенсивным развитием компьютерной техники и информационных технологий появилась важная сфера приложений теории массового обслуживания - проектирование и создание информационно-вычислительных сетей, компьютерных сетей связи, спутниковых сетей связи и т. п. Вполне естественно, что это дало толчок к необходимости построения новых математических моделей входящих потоков событий, достаточно адекватно описывающих реальные информационные потоки, функционирующие в таких сетях. Отметим, что одними из первых работ в этом направлении были статьи [1]-[3]. На практике параметры, определяющие входящий поток, изменяются со временем, при этом изменения часто носят случайный характер, что приводит к рассмотрению дважды стохастических потоков событий. Потоки с интенсивностью, являющейся случайным процессом, можно разделить на два класса. К первому классу относятся потоки с интенсивностью, являющейся непрерывным случайным процессом. Ко второму классу относятся потоки, у которых интенсивность есть кусочно-постоянный случайный процесс. Последние (потоки с переключениями или МС-потоки $[1,2])$ являются наиболее характерными для реальных телекоммуникационных сетей. В свою очередь, МС-потоки можно разделить на три типа:

(1) синхронные потоки - потоки с интенсивностью, для которой переход из состояния в состояние происходит в случайные моменты времени, являющиеся моментами наступления событий [4]; 
(2) асинхронные потоки - потоки с интенсивностью, для которой переход из состояния в состояние происходит в случайные моменты времени и не зависит от моментов наступления событий [5];

(3) полусинхронные потоки - потоки, которые в одном множестве состояний имеют тип 1 , а в остальных состояниях имеют тип 2 [6].

Подчеркнем, что отмеченные синхронные, асинхронные и полусинхронные потоки можно представить в виде моделей MAP (Markovian Arrival Process) потоков событий [7] с определенными ограничениями на параметры последних [8].

В настоящей работе рассматривается асинхронный поток с инициированием лишних событий, являющийся обобщением асинхронного потока.

\section{2. Постановка задачи}

Рассматривается асинхронный дважды стохастический поток с инициированием лишних событий (далее обобщенный асинхронный поток), интенсивность которого есть кусочнопостоянный стационарный процесс $\lambda(t)$ с двумя состояниями $\lambda_{1}$ и $\lambda_{2}, \lambda_{1}>\lambda_{2}$. В течение временного интервала, когда $\lambda(t)=\lambda_{i}$, имеет место пуассоновский поток событий с интенсивностью $\lambda_{i}, i=1,2$. Переход из первого состояния процесса $\lambda(t)$ во второе (как и из второго в первое) может осуществляться в произвольный момент времени. При этом длительность пребывания процесса $\lambda(t)$ в $i$-ом состоянии распределена по экспоненциальному закону с параметром $\alpha_{i}, i=1,2$. При переходе процесса $\lambda(t)$ из первого состояния во второе с вероятностью $p, 0 \leqslant p \leqslant 1$, инициируется лишнее событие во втором состоянии (т.е. сначала осуществляется переход, а затем инициируется лишнее событие). Наоборот, при переходе процесса $\lambda(t)$ из второго состояния в первое лишнее событие в первом состоянии инициируется с вероятностью $q, 0 \leqslant q \leqslant 1$. Очевидно, что в сделанных предпосылках $\lambda(t)$ - марковский процесс. Вариант возникающей ситуации показан на рис. 1. Если $p=q=0$, то имеет место асинхронный поток [5].

Обозначим $\tau_{i}=t_{i+1}-t_{i}, i=1,2, \ldots$, длительность $i$-го интервала между событиями потока. Пусть $p\left(\tau_{i}\right)$ - плотность вероятностей длительности $i$-го интервала; $p\left(\tau_{i}, \tau_{i+1}\right)$ - совместная плотность вероятностей длительностей двух смежных интервалов, $i=1,2, \ldots$ Рассмотрим стационарный режим функционирования потока. Тогда $p\left(\tau_{i}\right)=p(\tau), p\left(\tau_{i}, \tau_{i+1}\right)=p\left(\tau_{1}, \tau_{2}\right)$ для любого $i$, где $\tau-$ длительность интервала, произвольно расположенного на временной оси, $\tau_{1}, \tau_{2}$-длительности двух смежных интервалов, также произвольно расположенных на временной оси.

\section{3. Вывод явной формулы для $p(\tau)$}

В обобщенном асинхронном потоке присутствуют четыре типа событий: 1-й (2-й) тип - события пуассоновского потока с параметром $\lambda_{1}\left(\lambda_{2}\right)$; 3-й (4-й) тип - лишние события, инициированные с вероятностью $p(q)$ во втором состоянии (в первом состоянии) процесса $\lambda(t)$ при его переходе из первого состояния во второе (из второго состояния в первое). Пусть $\tau=0$ - момент наступления события.

Пусть $\pi_{i j}(\tau)$ - условная вероятность того, что на интервале $(0, \tau)$ нет событий потока и $\lambda(\tau)=\lambda_{j}, j=1,2$, при условии, что в момент $\tau=0$ имело место событие $i$-го типа, $i=1, \ldots, 4$. Для вероятностей $\pi_{i j}(\tau)$ нетрудно выписать следующие системы 


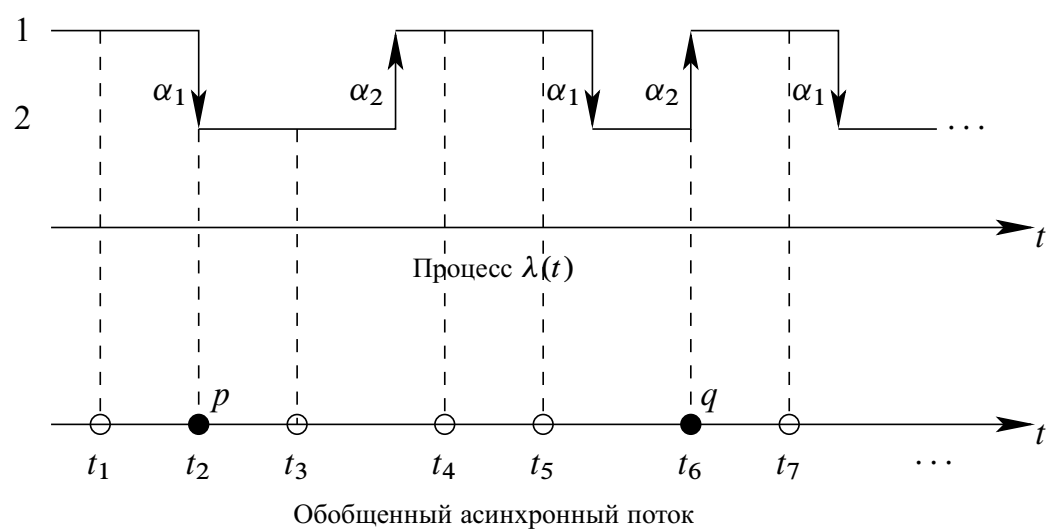

Рис. 1. Формирование обобщенного асинхронного потока: 1,2 - состояния случайного процесса $\lambda(t) ; t_{1}, t_{2}, \ldots$ - моменты наступления событий; $t_{2}, t_{6}, \ldots-$ моменты инициирования лишних событий; $t_{2}-$ момент инициирования с вероятностью $p$ лишнего события во втором состоянии; $t_{6}-$ момент инициирования с вероятностью $q$ лишнего события в первом состоянии

дифференциальных уравнений:

$$
\begin{aligned}
& \pi_{i 1}^{\prime}(\tau)=-\left(\lambda_{1}+\alpha_{1}\right) \pi_{i 1}(\tau)+(1-q) \alpha_{2} \pi_{i 2}(\tau), \\
& \pi_{i 2}^{\prime}(\tau)=-\left(\lambda_{2}+\alpha_{2}\right) \pi_{i 2}(\tau)+(1-p) \alpha_{1} \pi_{i 1}(\tau), \quad i=1, \ldots, 4,
\end{aligned}
$$

с граничными условиями

$$
\begin{aligned}
& \pi_{11}(0)=\pi_{22}(0)=\pi_{32}(0)=\pi_{41}(0)=1, \\
& \pi_{12}(0)=\pi_{21}(0)=\pi_{31}(0)=\pi_{42}(0)=0 .
\end{aligned}
$$

Решая (1), находим

$$
\begin{aligned}
& \pi_{11}(\tau)=\pi_{41}(\tau)=-\frac{\lambda_{1}+\alpha_{1}-z_{2}}{z_{2}-z_{1}} e^{-z_{1} \tau}+\frac{\lambda_{1}+\alpha_{1}-z_{1}}{z_{2}-z_{1}} e^{-z_{2} \tau}, \\
& \pi_{12}(\tau)=\pi_{42}(\tau)=\frac{\alpha_{1}(1-p)}{z_{2}-z_{1}} e^{-z_{1} \tau}-\frac{\alpha_{1}(1-p)}{z_{2}-z_{1}} e^{-z_{2} \tau}, \\
& \pi_{21}(\tau)=\pi_{31}(\tau)=\frac{\alpha_{2}(1-q)}{z_{2}-z_{1}} e^{-z_{1} \tau}-\frac{\alpha_{2}(1-q)}{z_{2}-z_{1}} e^{-z_{2} \tau}, \\
& \pi_{22}(\tau)=\pi_{32}(\tau)=\frac{\lambda_{1}+\alpha_{1}-z_{1}}{z_{2}-z_{1}} e^{-z_{1} \tau}-\frac{\lambda_{1}+\alpha_{1}-z_{2}}{z_{2}-z_{1}} e^{-z_{2} \tau},
\end{aligned}
$$

где

$$
\begin{aligned}
z_{1,2}=\frac{1}{2}\left[\left(\lambda_{1}+\alpha_{1}+\lambda_{2}+\alpha_{2}\right)\right. & \\
& \left.\mp \sqrt{\left(\lambda_{1}-\lambda_{2}+\alpha_{1}-\alpha_{2}\right)^{2}+4 \alpha_{1} \alpha_{2}(1-p)(1-q)}\right], \quad 0<z_{1}<z_{2} .
\end{aligned}
$$

Пусть $\pi_{i j}-$ вероятность того, что за время, которое пройдет от момента $\tau=0$, в который имело место событие $i$-го типа, поведение потока будет таковым, что следующим событием будет событие $j$-го типа, $i, j=1, \ldots, 4$. При этом вероятность наступления 
события 1-го типа на полуинтервале $[\tau, \tau+\Delta \tau)$, где $\Delta \tau$ здесь и далее достаточно малая величина, есть $\lambda_{1} \Delta \tau+o(\Delta \tau)$; 2-го типа $-\lambda_{2} \Delta \tau+o(\Delta \tau)$; 3-го типа $-p \alpha_{1} \Delta \tau+o(\Delta \tau)$; 4-го типа $-q \alpha_{2} \Delta \tau+o(\Delta \tau)$. Тогда, с учетом введенных вероятностей $\pi_{i j}(\tau)$, получаем

$$
\begin{aligned}
& \pi_{i 1}=\lambda_{1} \int_{0}^{\infty} \pi_{i 1}(\tau) d \tau, \quad \pi_{i 2}=\lambda_{2} \int_{0}^{\infty} \pi_{i 2}(\tau) d \tau, \\
& \pi_{i 3}=p \alpha_{1} \int_{0}^{\infty} \pi_{i 1}(\tau) d \tau, \quad \pi_{i 4}=q \alpha_{2} \int_{0}^{\infty} \pi_{i 2}(\tau) d \tau, \quad i=1, \ldots, 4 .
\end{aligned}
$$

Подставляя (2) в (3), находим

$$
\begin{array}{lll}
\pi_{11}=\pi_{41}=\frac{\lambda_{1}\left(\lambda_{2}+\alpha_{2}\right)}{z_{1} z_{2}}, & \pi_{12}=\pi_{42}=\frac{(1-p) \lambda_{2} \alpha_{1}}{z_{1} z_{2}}, \\
\pi_{13}=\pi_{43}=\frac{p \alpha_{1}\left(\lambda_{2}+\alpha_{2}\right)}{z_{1} z_{2}}, & \pi_{14}=\pi_{44}=\frac{q(1-p) \alpha_{1} \alpha_{2}}{z_{1} z_{2}}, \\
\pi_{21}=\pi_{31}=\frac{(1-q) \lambda_{1} \alpha_{2}}{z_{1} z_{2}}, & \pi_{22}=\pi_{32}=\frac{\lambda_{2}\left(\lambda_{1}+\alpha_{1}\right)}{z_{1} z_{2}}, \\
\pi_{23}=\pi_{33}=\frac{p(1-q) \alpha_{1} \alpha_{2}}{z_{1} z_{2}}, & \pi_{24}=\pi_{34}=\frac{q \alpha_{2}\left(\lambda_{1}+\alpha_{1}\right)}{z_{1} z_{2}} .
\end{array}
$$

Пусть $q_{i}-$ стационарная вероятность того, что наступившее событие есть событие $i$-го типа, $i=1, \ldots, 4$. Нетрудно показать, что последовательность моментов наступления событий $t_{1}, t_{2}, \ldots$ (см. рис. 1) образует вложенную цепь Маркова [9]. Тогда для вероятностей $q_{i}, i=1, \ldots, 4$, имеет место следующая система уравнений:

$$
\begin{aligned}
\sum_{i=1}^{4} \pi_{i j} q_{i} & =q_{j}, \quad j=1, \ldots, 4, \\
\sum_{i=1}^{4} q_{i} & =1,
\end{aligned}
$$

где $\pi_{i j}$ определены в (4). Решая (5), получаем

$$
q_{1}=\lambda_{1} \alpha_{2} a, \quad q_{2}=\lambda_{2} \alpha_{1} a, \quad q_{3}=p \alpha_{1} \alpha_{2} a, \quad q_{4}=q \alpha_{1} \alpha_{2} a,
$$

где

$$
a=\left[\lambda_{1} \alpha_{2}+\lambda_{2} \alpha_{1}+(p+q) \alpha_{1} \alpha_{2}\right]^{-1} .
$$

Пусть $\tilde{\pi}_{i}-$ условная стационарная вероятность того, что процесс $\lambda(\tau)$ в момент времени $\tau=0$ находится в $i$-ом состоянии при условии, что в этот момент наступило событие потока, $i=1,2$. Тогда, учитывая (6), находим

$$
\begin{aligned}
& \tilde{\pi}_{1}=q_{1}+q_{4}=\alpha_{2}\left(\lambda_{1}+q \alpha_{1}\right) a, \\
& \tilde{\pi}_{2}=q_{2}+q_{3}=\alpha_{1}\left(\lambda_{2}+p \alpha_{2}\right) a .
\end{aligned}
$$

Заметим, что вероятность $\tilde{\pi}_{i}, i=1,2$, отличается от безусловной вероятности $\pi_{i}$ того, что процесс $\lambda(\tau)$ в произвольный момент времени находится в $i$-ом состоянии [5]:

$$
\pi_{1}=\frac{\alpha_{2}}{\alpha_{1}+\alpha_{2}}, \quad \pi_{2}=\frac{\alpha_{1}}{\alpha_{1}+\alpha_{2}}, \quad \pi_{1}+\pi_{2}=1
$$


Пусть $p_{i j}(\tau)$ - условная вероятность того, что на интервале $(0, \tau)$ нет событий потока и $\lambda(\tau)=\lambda_{j}$ при условии, что в момент $\tau=0$ событие произошло и $\lambda(0)=\lambda_{i}, i, j=1,2$. Вероятности $p_{i j}(\tau)$ для $i=1,2$ определяются теми же системами дифференциальных уравнений $(1)$, что и вероятности $\pi_{i j}(\tau)$, с теми же граничными условиями. Вследствие этого, для вероятностей $p_{i j}(\tau)$ справедливы формулы $(2)$, в которых вместо $\pi_{i j}(\tau)$ нужно поставить $p_{i j}(\tau), i, j=1,2$. Тогда искомую плотность $p(\tau)$ можно записать в виде

$$
p(\tau)=\sum_{i=1}^{2} \tilde{\pi}_{i} \sum_{j=1}^{2}\left(\lambda_{j}+p_{j} \alpha_{j}\right) p_{i j}(\tau),
$$

где $p_{1}=p, p_{2}=q$. Подставляя (2), (7) в (8), получаем

$$
\begin{aligned}
p(\tau) & =\gamma z_{1} e^{-z_{1} \tau}+(1-\gamma) z_{2} e^{-z_{2} \tau}, \quad \tau \geqslant 0, \\
\gamma & =\frac{1}{z_{2}-z_{1}}\left[z_{2}-\lambda_{1}-\lambda_{2}+\frac{\left(\alpha_{1}+\alpha_{2}\right)\left(\lambda_{1} \lambda_{2}-\alpha_{1} \alpha_{2} p q\right)}{\lambda_{1} \alpha_{2}+\lambda_{2} \alpha_{1}+(p+q) \alpha_{1} \alpha_{2}}\right] .
\end{aligned}
$$

\section{4. Вывод явной формулы для $p\left(\tau_{1}, \tau_{2}\right)$}

Пусть $\tau_{1} \geqslant 0, \tau_{2} \geqslant 0$ - длительности двух смежных интервалов; их расположение на временной оси, в силу стационарности потока, произвольно. Так как последовательность моментов наступления событий есть вложенная цепь Маркова, то совместная плотность вероятностей $p\left(\tau_{1}, \tau_{2}\right)$ принимает вид

$$
\begin{aligned}
p\left(\tau_{1}, \tau_{2}\right)=\sum_{i=1}^{2} \tilde{\pi}_{i} \sum_{j=1}^{2} a_{j} p_{i j}\left(\tau_{1}\right) \sum_{k=1}^{2}\left(a_{k}+b_{k}\right) p_{1 k}\left(\tau_{2}\right) & \\
& +\sum_{i=1}^{2} \tilde{\pi}_{i} \sum_{j=1}^{2} b_{j} p_{i j}\left(\tau_{1}\right) \sum_{k=1}^{2}\left(a_{k}+b_{k}\right) p_{2 k}\left(\tau_{2}\right),
\end{aligned}
$$

где $a_{1}=\lambda_{1}, a_{2}=q \alpha_{2}, b_{1}=p \alpha_{1}, b_{2}=\lambda_{2}$, и $\tilde{\pi}_{i}$ определены формулами (7), $p_{i j}\left(\tau_{k}\right)-$ формулами (2) для $\tau=\tau_{1}$ либо $\tau=\tau_{2}, i, j, k=1,2$. Подставляя их выражения в (10), находим

$$
\begin{aligned}
p\left(\tau_{1}, \tau_{2}\right) & =p\left(\tau_{1}\right) p\left(\tau_{2}\right) \\
& +\gamma(1-\gamma) \frac{\lambda_{1} \lambda_{2}-p q \alpha_{1} \alpha_{2}}{z_{1} z_{2}}\left(z_{1} e^{-z_{1} \tau_{1}}-z_{2} e^{-z_{2} \tau_{1}}\right)\left(z_{1} e^{-z_{1} \tau_{2}}-z_{2} e^{-z_{2} \tau_{2}}\right),
\end{aligned}
$$

где $\gamma, p\left(\tau_{j}\right)$ определены в (9) для $\tau=\tau_{j}, j=1,2$.

Из (11) следует, что обобщенный асинхронный поток в общем случае является коррелированным потоком.

Нетрудно получить вероятностные характеристики потока, такие, как математическое ожидание длительности интервала между событиями, дисперсию и ковариацию:

$$
\begin{gathered}
\mathbf{M} \tau=\frac{\gamma}{z_{1}}+\frac{1-\gamma}{z_{2}}, \quad \quad \mathbf{D} \tau=2\left(\frac{\gamma}{z_{1}^{2}}+\frac{1-\gamma}{z_{2}^{2}}\right)-\left(\frac{\gamma}{z_{1}}+\frac{1-\gamma}{z_{2}}\right)^{2}, \\
\operatorname{cov}\left(\tau_{1}, \tau_{2}\right)=\gamma(1-\gamma)\left(z_{1}-z_{2}\right)^{2} \frac{\lambda_{1} \lambda_{2}-p q \alpha_{1} \alpha_{2}}{\left(z_{1} z_{2}\right)^{3}},
\end{gathered}
$$


где $z_{1}, z_{2}$ определены в (2), а $\gamma$ в (9).

Рассмотрим частные случаи, при которых обобщенный асинхронный поток становится рекуррентным либо вырождается в простейший. Для ситуаций:

(1) $\lambda_{1} \lambda_{2}=p q \alpha_{1} \alpha_{2}$

(2) $\lambda_{2}=0, p=0$,

(3) $\lambda_{2}=0, q=0$

совместная плотность (11) факторизуется. Так как последовательность моментов наступления событий есть вложенная цепь Маркова, то нетрудно показать, что факторизуется и совместная плотность $p\left(\tau_{1}, \ldots, \tau_{n}\right)$. Последнее означает, что для этих ситуаций обобщенный асинхронный поток является рекуррентным [9].

Если $\gamma(1-\gamma)=0$ (это равенство имеет место, если либо $\lambda_{1}+p \alpha_{1}=\lambda_{2}+q \alpha_{2}$ либо $\left.\lambda_{1}+q \alpha_{1}=\lambda_{2}+p \alpha_{2}\right)$, то обобщенный асинхронный поток вырождается в простейший с параметром $\lambda_{1}+p \alpha_{1}$ либо с параметром $\lambda_{1}+q \alpha_{1}$.

Наконец, рассмотрим особый случай, когда $z_{1}=z_{2}$, при котором в формулах (9), (11) реализуется деление на ноль. Этот случай имеет место, если $\lambda_{1}+\alpha_{1}=\lambda_{2}+\alpha_{2}$ и $q=1$ (либо $p=1$ ). Здесь

$$
\begin{aligned}
p(\tau)= & \left(\lambda_{1}+\alpha_{1}\right) \frac{\alpha_{1}\left(\lambda_{2}+p \alpha_{2}\right)+\alpha_{2}\left(\lambda_{1}+p \alpha_{1}\right)+(1-p) \alpha_{1} \alpha_{2}\left(\lambda_{1}+\alpha_{1}\right) \tau}{\lambda_{1} \alpha_{2}+\lambda_{2} \alpha_{1}+(1+p) \alpha_{1} \alpha_{2}} \\
& \times e^{-\left(\lambda_{1}+\alpha_{1}\right) \tau}, \quad \tau \geqslant 0, \\
p\left(\tau_{1}, \tau_{2}\right)= & p\left(\tau_{1}\right) p\left(\tau_{2}\right)-\frac{(1-p)^{2} \alpha_{1}^{2} \alpha_{2}^{2}\left(\lambda_{1} \lambda_{2}-p \alpha_{1} \alpha_{2}\right)}{\left[\lambda_{1} \alpha_{2}+\lambda_{2} \alpha_{1}+(1+p) \alpha_{1} \alpha_{2}\right]^{2}} \\
& \times\left[1-\left(\lambda_{1}+\alpha_{1}\right) \tau_{1}\right]\left[1-\left(\lambda_{1}+\alpha_{1}\right) \tau_{2}\right] e^{-\left(\lambda_{1}+\alpha_{1}\right)\left(\tau_{1}+\tau_{2}\right)} .
\end{aligned}
$$

В (13) $p\left(\tau_{j}\right)$ определены формулой (12) для $\tau=\tau_{j}, j=1,2$. Из (13) вытекает, что обобщенный асинхронный поток для особого случая также является коррелированным потоком. Если же $\lambda_{1} \lambda_{2}=p \alpha_{1} \alpha_{2}$, то поток становится рекуррентным; если $p=1$, то поток вырождается в простейший с параметром $\lambda_{1}+\alpha_{1}$. Для ситуации $\lambda_{1}+\alpha_{1}=\lambda_{2}+\alpha_{2}$ и $p=1$ в формулах (12), (13) нужно вместо $p$ подставить $q$.

\section{5. Заключение}

Реальные ситуации в телекоммуникационных сетях характеризуются тем, что параметры, определяющие входящий поток событий, либо частично известны, либо, вообще неизвестны. В связи с этим возникают два класса задач:

(1) задача фильтрации интенсивности потока (или задача оценивания состояний потока);

(2) задача оценивания параметров потока.

При решении этих задач полезна информация о свойствах потока, в частности, при каких условиях коррелированный поток становится рекуррентным. 


\section{Список литературы}

1. Башарин Г. П., Кокотушкин В. А., Наумов В. А., О методе эквивалентных замен расчета фрагментов сетей связи. Известия АН СССР, сер. Техн. киберн. (1979), № 6, 92-99.

2. Башарин Г. П., Кокотушкин В. А., Наумов В. А., О методе эквивалентных замен расчета фрагментов сетей связи. Известия АН СССР, сер. Техн. киберн. (1980), № 1, 55-61.

3. Neuts M. F., A versatile Markov point process. J. Appl. Probab. (1979) 16, 764-779.

4. Бушланов И. В., Горцев А. М., Оптимальная оценка состояний синхронного дважды стохастического потока событий. Автоматика и телемеханика (2004), № 9, 40-51.

5. Васильева Л. А., Горцев А. М., Оценивание длительности мертвого времени асинхронного дважды стохастического потока событий в условиях его неполной наблюдаемости. Автоматика и телемеханика (2003), № 12, 69-79.

6. Горцев А. М., Нежельская Л. А., Оценивание периода мертвого времени и параметров полусинхронного дважды стохастического потока событий. Измерительная техника (2003), №6, $7-13$.

7. Lucantoni D. M., Neuts M. F., Some steady-state distributions for the $M A P / S M / 1$ queue. Comm. Stat., Stochastic Models (1994) 10, 575-598.

8. Василевская Т. П., Завгородняя М. Е., Шмырин И. С., О соотношении моделей МАР-потока событий и асинхронного, полусинхронного и синхронного дважды стохастических потоков событий. Вестник Томского государственного университета (2004), № 9 (II), 138-144.

9. Ивченко Г. И., Каштанов В. А., Коваленко И. Н., Теория массового обслуживания. Высшая школа, Москва, 1982.

Статья поступила 14.12.2007. 